\title{
Correction: Discovery of a FLT3 inhibitor LDD1937 as an anti- leukemic agent for acute myeloid leukemia
}

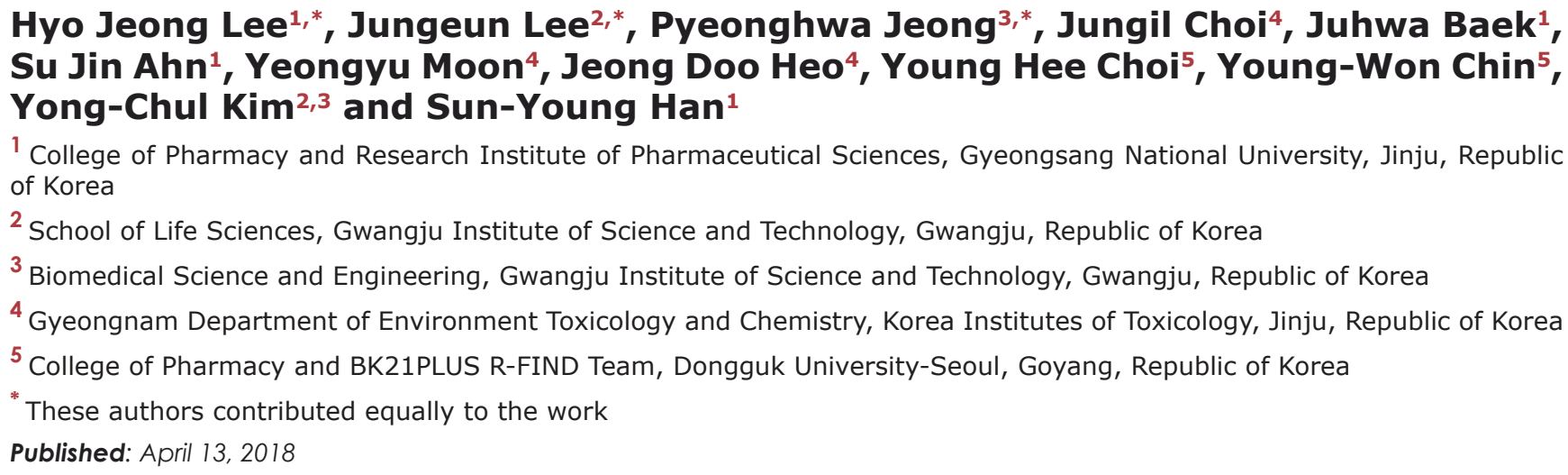
Su Jin Ahn', Yeongyu Moon ${ }^{4}$, Jeong Doo Heo ${ }^{4}$, Young Hee Choi ${ }^{5}$, Young-Won Chin ${ }^{5}$, Yong-Chul Kim ${ }^{2,3}$ and Sun-Young Han ${ }^{1}$

${ }^{1}$ College of Pharmacy and Research Institute of Pharmaceutical Sciences, Gyeongsang National University, Jinju, Republic of Korea

${ }^{2}$ School of Life Sciences, Gwangju Institute of Science and Technology, Gwangju, Republic of Korea

${ }^{3}$ Biomedical Science and Engineering, Gwangju Institute of Science and Technology, Gwangju, Republic of Korea

${ }^{4}$ Gyeongnam Department of Environment Toxicology and Chemistry, Korea Institutes of Toxicology, Jinju, Republic of Korea

${ }^{5}$ College of Pharmacy and BK21PLUS R-FIND Team, Dongguk University-Seoul, Goyang, Republic of Korea

* These authors contributed equally to the work

Published: April 13, 2018

Copyright: Lee et al. This is an open-access article distributed under the terms of the Creative Commons Attribution License 3.0 (CC BY 3.0), which permits unrestricted use, distribution, and reproduction in any medium, provided the original author and source are credited.

This article has been corrected: The correct author affiliations citations are given below:

\section{Hyo Jeong Lee ${ }^{1, *}$, Jungeun Lee ${ }^{2, *}$, Pyeonghwa Jeong ${ }^{3, *}$, Jungil Choi ${ }^{4}$, Juhwa Baek ${ }^{1}$, Su Jin Ahn ${ }^{1}$, Yeongyu Moon ${ }^{4}$, Jeong Doo Heo ${ }^{4}$, Young Hee Choi ${ }^{5}$, Young-Won Chin ${ }^{5}$, Yong-Chul Kim ${ }^{2,3}$ and Sun-Young Han ${ }^{1}$}

Original article: Oncotarget. 2018; 9:924-936. https://doi.org/10.18632/oncotarget.23221 